\title{
Annexin A2 extracellular translocation and virus interaction: a potential target for antivirus-drug discovery
}

\begin{abstract}
Annexin A2 is a membrane scaffolding and binding protein, which mediated various cellular events. Its functions are generally affected by cellular localization. In the cytoplasm, they interacted with different phospholipid membranes in $\mathrm{Ca} 2+-$ dependent manner and play vital roles including actin binding, remodeling and dynamics, cytoskeletal rearrangement, and lipidraft microdomain formation. However, upon cell exposure to certain stimuli, annexin A2 translocates to the external leaflets of the plasma membrane where annexin A2 was recently reported to serve as a virus receptor, play an important role in the formation of virus replication complex, or implicated in virus assembly and budding. Here, we review some of annexin A2 roles in virus infections and the potentiality of targeting annexin A2 in the design of novel and promising antivirus agent that may have a broader consequence in virus therapy.
\end{abstract}

Keyword: Annexin A2; Drug-target; Translocation; Virus-interaction 